\title{
Mathematical Modeling and Interpretation of PbPc Gas Sensor Array
}

\author{
${ }^{1}$ Nidal F. Shilbayeh and ${ }^{2}$ Mahmoud Z. Iskandarani \\ ${ }^{1}$ Faculty of Information Technology for Graduate Studies, The university of Graduate Studies \\ P.O. Box 42, Post Code 111610, Amman, Jordan \\ ${ }^{2}$ Faculty of Science and Information Technology, Al-Zaytoonah Private University of Jordan \\ P.O. Box 911597, Post Code 11191, Amman, Jordan
}

\begin{abstract}
The voltage/current characteristics and the effect of $\mathrm{NO}_{2}$ gas on the electrical conductivity of a $\mathrm{PbPc}$ gas sensor array is investigated. The gas sensor is manufactured using vacuum deposition of gold electrodes on sapphire substrate with the lead-phathalocyanine vacuum sublimed on the top of the gold electrodes. Two versions of the PbPc gas sensor array are investigated. The tested types differ in the gap sizes between the deposited gold electrodes. The sensors are tested at different temperatures to account for conductivity changes as the molecular adsorption/desorption rate is affected by heat. The obtained results found to be encouraging as the sensors showed stability and sensitivity towards low concentration of applied $\mathrm{NO}_{2}$ gas.
\end{abstract}

Key words: Intelligent system, PbPc, gas sensor, hardware, software, algorithm

\section{INTRODUCTION}

The gases absorbed on the surfaces of the organic materials have a marked effect on the electrical conductivity of these materials; where the electrical conductivity of an organic material that contains transition and heavy central atoms changes by many orders of magnitude when a gas adsorbs on its surface.

Adsorption involves the formation of bonds between the adsorbed gas and the organic material, by transfer of electrical charge The charge transfer changes the electronic structure of the material; changing its conductivity. Changes in the conductivity are related to the number of gas molecules adsorbed on the surface, and hence, to the concentration of the adsorbed species in the surrounding atmosphere. The measurement of changes in electrical conductance due to gas adsorption makes organic materials ideal for the detection of low gas concentration.

The conductivity of an organic material can either be affected at the surface with no bulk interaction, or following the reaction at the surface; carriers can be injected into the bulk causing both surface and bulk conductivities to be affected ${ }^{[1-3]}$.

When a gas molecule chemisorbs on the surface of the organic material, an electron or electrons may be transferred from one to the other, where the direction of transfer depends on the electro negativity of the gas and the work function of the solid. Also the physisorption of the gas plays an important role in the overall mechanism of gas adsorption and subsequent conductivity changes in the organic material. The adsorption of the gas molecules as ions on the surface removes electrons from the solid and localizes them at the surface, thus generating electron traps in the solid. This results in an increase in the hole concentration and therefore an increase in the conductance as the material will function as a P-type material.

In this paper the properties of the $\mathrm{PbPc}$ sensor array is confirmed, modeled, and employed in an intelligent discriminating and controlling system. The system uses the inter-digital geometry together with $\mathrm{PbPc}$ film properties to distinguish between types of gases.

\section{Background}

Metal-phthalocyanines: They are a group of organic materials known to have several properties that make them attractive as a potential gas sensing materials or gas detectors. They have the following desirable properties:

1. They are considered to be good electrical conductors, where the charge carriers are known to be positive holes ${ }^{[4-6]}$.

2. Reaction of Phthalocyanines with electron accepting gases should increase its conductivity.

3. They are thermally stable to temperatures in excess of 400 degrees centigrade.

4. They can be used for extended periods at elevated temperatures.

5. Organic groups can be easily modified and functional groups can be substituted on either ring structure or on the central hydrogen atom in the metal-free Phthalocyanines $\left(\mathrm{H}_{2} \mathrm{Pc}\right)$ as shown in Fig. 1.

Several of the Metal-Phthalocyanine materials (e.g. commercial materials) contain significant amounts of impurities which can result in irreproducible electrical characteristics, which can be purified using either Gas-

Corresponding Author: Faculty of Information Technology for Graduate Studies, The university of Graduate Studies P.O. Box 42, Post Code 111610, Amman, Jordan 


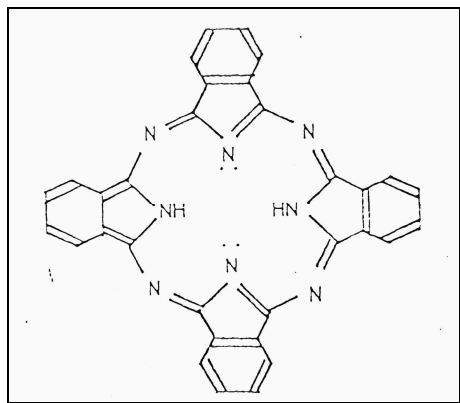

Fig. 1: $\mathrm{H}_{2} \mathrm{Pc}$ molecular representation

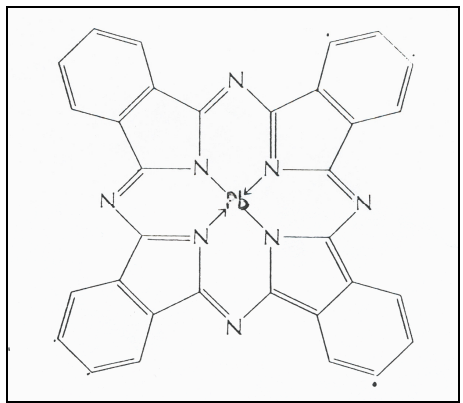

Fig. 2: $\mathrm{PbPc}$ molecular representation

Entrainer Sublimation (in Nitrogen or Oxygen) or by Vacuum Sublimation (the used technique in our work). The purification process leads to stable and reproducible electrical characteristics. Early studies of Phthalocyanines showed that many are sensitive to the presence of $\mathrm{NO}_{2}$ with an increase in the sensitivity observed when the Phthalocyanines contains heavier central atoms.

Metal-Phthalocyanines organic semiconductors found to undergo conductivity changes upon the adsorption of strongly electrophilic gases such as $\mathrm{NO}_{2}$, $\mathrm{CL}_{2}$ and $\mathrm{F}_{2}$. The magnitude and reversibility of these conductivity variations found to be dependant on the central metal species. Because of their high decomposition temperatures (450 Degrees Centigrade), Phthalocyanines can be vacuum evaporated to produce thin films. The deposition of such films on the surface of a substrate eliminates some of the complications arising from having to consider both surface and bulk conductivities in the analysis process. The absence of bulk conductivity effect will improve the response and recovery times of the designed sensor array. However, the presence of impurities will affect resistivity, linearity, hysterisis and drift of the electrical characteristics. Also the conduction activation energy and the specific conduction mechanism will be affected.

Lead-phthalocyanines (PbPc): It is the most sensitive and stable Phthalocyanine material. Although, insensitive to a wide range of gases (e.g. weak electrophilic and electrophobic gases and $\mathrm{CH}_{4}, \mathrm{CO}, \mathrm{O}_{2}$, $\mathrm{H}_{2} \mathrm{O}, \mathrm{H}_{2}$, Hydrocarbons and changes in $\mathrm{O}_{2}$ pressure), $\mathrm{PbPc}$ based sensor is very sensitive and potentially useful in detecting strong electrophilic gases

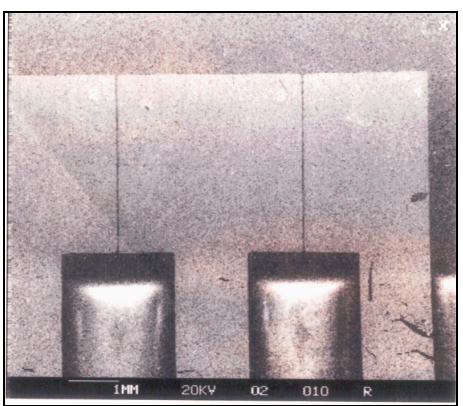

Fig. 3: 10:10:10 gap separation

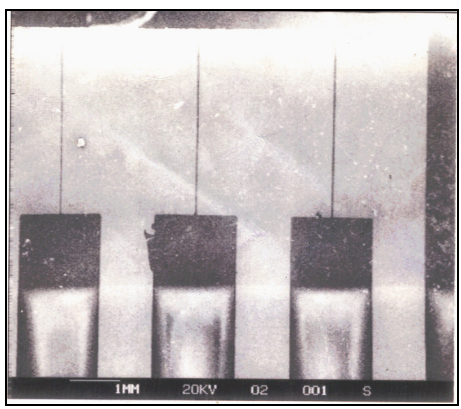

Fig. 4: 5:10:15 gap separation

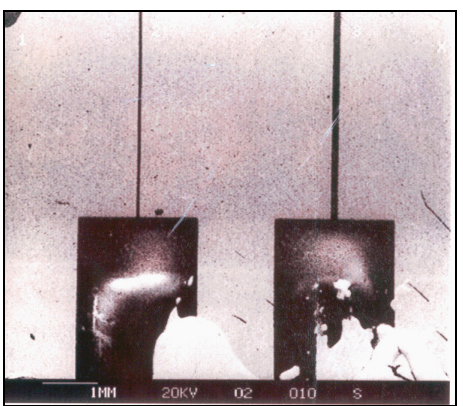

Fig. 5: 10:33 gap separation

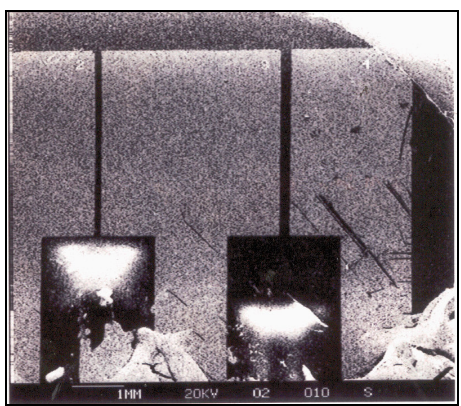

Fig. 6: $33: 100$ gap separation

particularly to $\mathrm{NO}_{2}$ or $\mathrm{NO}_{\mathrm{x}}$ and will respond to low concentrations of $\mathrm{F}_{2}$ and less than $1 \mathrm{ppm}$ concentration of $\mathrm{CL}_{2}$ gases. Thus it could form the basis of a highly sensitive and selective $\mathrm{NO}_{2}$ or $\mathrm{NO}_{\mathrm{x}}$ sensor and it is suitable for detecting these gases at concentrations from $1 \mathrm{ppb}$ to $10 \mathrm{ppm}$ in air and retains discrimination to concentrations above 100ppm.

Since $\mathrm{PbPC}$ has high sensitivity and selectivity to strongly accepting gases such as $\mathrm{NO}_{2}, \mathrm{CL}_{2}, \mathrm{~F}_{2}$, and $\mathrm{O}_{3}$, discrimination between strongly accepting gases and 
other gases is achieved by careful choice of operating temperatures. However, the stability and electrical properties of this type of sensors is affected by water vapor, so a heating element is usually fitted to the back of the sensor to eliminate such factor.

It is found ${ }^{[6]}$ that $\mathrm{PbPc}$ sensor is most sensitive to $\mathrm{NO}_{2}$ gas and can be operated in a continuous mode at temperatures above 100 degrees $\mathrm{C}$ with its sensitivity and stability decreasing as the temperature approaches 200 Degrees C. These temperatures are also a function of PbPc film thickness deposited on the substrate and can be extended when thick films are used.

PbPC sensor design: The sensor is based on a sapphire substrate (Alpha- $\mathrm{Al}_{2} \mathrm{O}_{3}$ ), which usually contains traces of $\mathrm{Fe}$ and $\mathrm{Ti}$ ions. The substrate has a very high melting point (about 2050 Degrees C). It is very hard and quite inert, especially towards acids. The substrate has an area of $12.5 \mathrm{~mm}$ by $12.5 \mathrm{~mm}$. Different types of electrode gap separations are produced to study the effect of inter-electrode separation of sensor sensitivity as shown in SEM photographs in Fig. 3-6. The sensor is mounted on a transistor like base, as it comprises three gaps.

\section{MATERIALS AND METHODS}

A testing chamber is built with air and temperature controls supplied through a computer controlled unit. Electrical characteristics of the sensor and its response to gases are recorded via specially designed hardware/software system as shown in Fig. $7^{[7-10]}$.

Sensor interface circuit: The obtained signals from the $\mathrm{PbPc}$ sensor array is known to have relatively small values, hence, a differential amplifier interface circuit is designed and used to condition the sensor output signal to the remaining part of the signal processing system, which also includes A/D converter and noise filters. The basic amplifying unit is shown in Fig. $8^{[11-13]}$.

In Fig. 8 each two inter electrodes produce a voltage signal proportional to the gap size and film thickness of the deposited PbPc film. The difference in the voltage of each two electrodes is amplified and processed to the next stage. The amplifying unit acts as an impedance matcher, so that a correct measure of the detected gas level is acquired with minimal signal loss.

Interfacing Algorithm: After digitizing the amplified and cleaned sensor array signals, they are processed to be analyzed using the algorithm shown in Fig. 9.

Mathematical modeling: To model the behavior of our multi-gap sensor, a form of linear transformation is used to transform a unit circle of the Z-plane to the upper half $\left(I_{m}>0\right)$ of the W-plane as shown in Fig. 10a and $b$.

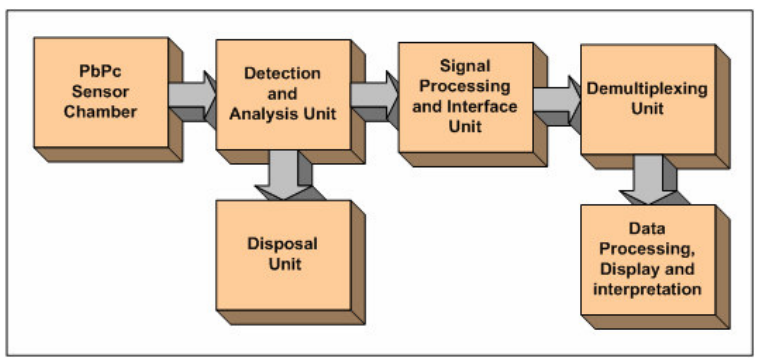

Fig. 7: Testing system

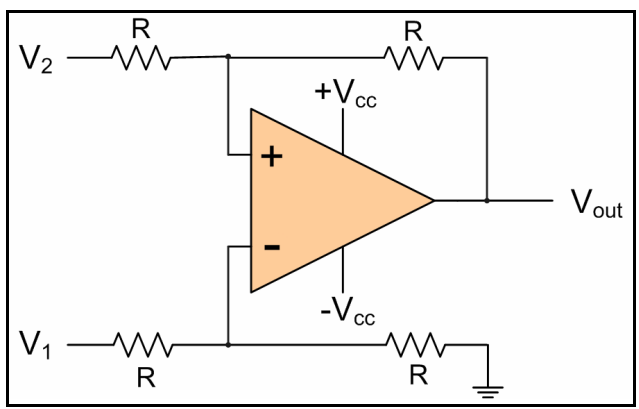

Fig. 8: Differential amplifier interface unit

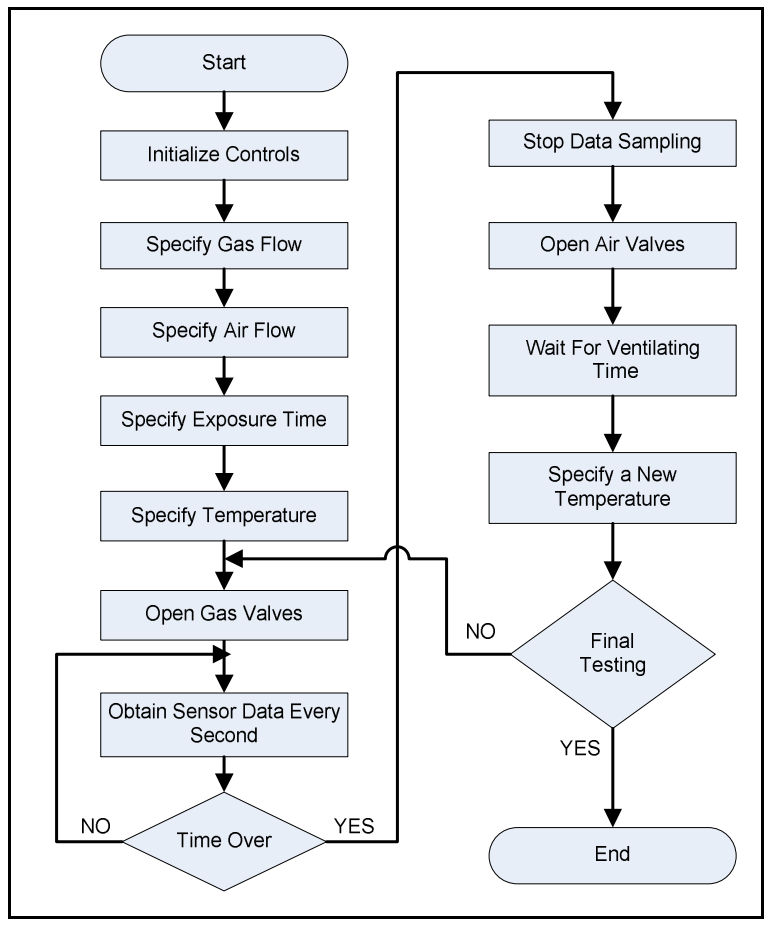

Fig. 9: Interface algorithm

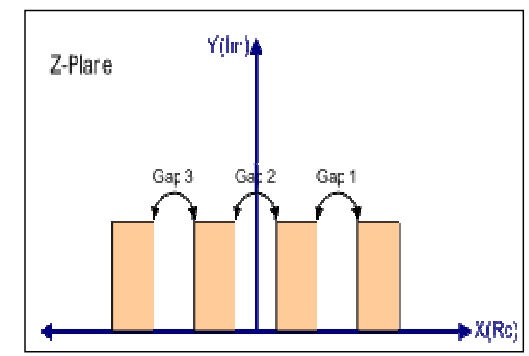

Fig. 10a: Inter electrode gap separations 
Fig. 10b: Inter electrode angle

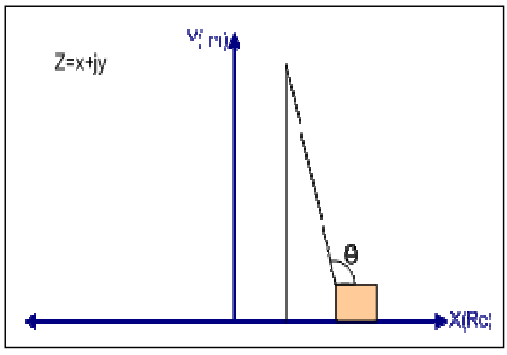

Consider the transformation:

$z=x+j y=f(t)=j \cdot\left(\frac{1-t}{1+t}\right) \quad \cdots$

So,

$t=-\left(\frac{z-j}{z+j}\right)$

Now, if $\left(x_{1}\right)$ is any general point on the $\mathrm{X}$-axis of the Z-plane and if it is represented in its polar form with a corresponding angle of $\left(\theta_{1}\right)$ then, equation (2) can be written as:

$e^{j \theta_{1}}=-\left(\frac{x_{1}-j}{x_{1}+j}\right) \cdots$

So,

$\theta_{1}=j \ln \left(\frac{x_{1}-j}{x_{1}+j}\right) \cdots$

Whence,

$d \theta_{1}=j\left(\frac{x_{1}+j}{x_{1}-j}\right)\left(\frac{-2 d x_{1}}{\left(x_{1}+j\right)^{2}}\right)=\left(\frac{2 d x_{1}}{\left(1+x_{1}^{2}\right)}\right) \cdots$

Now, the SCHWARZ complex potential solution $W=\theta+j \psi \quad$ is obtained through the following evaluation:

$W=\frac{1}{2 \pi} \int_{-\infty}^{+\infty}\left(\frac{e^{j \theta_{1}}+t}{e^{j \theta_{1}}-t}\right) \varphi\left(x_{1}\right) d \theta_{1} \cdots$

Now substituting equation (2), (3), and (4) into (6) gives:

$W=\frac{1}{2 \pi} \int_{-\infty}^{+\infty}\left(\frac{-\left(1+x_{1} z\right)}{j\left(z-x_{1}\right)}\right)\left(\frac{2 d x_{1}}{\left(1+x_{1}^{2}\right)}\right) \varphi\left(x_{1}\right) \cdots$

$W=\frac{j}{\pi} \int_{-\infty}^{+\infty}\left(\frac{\left(1+x_{1} z\right)}{\left(1+x_{1}^{2}\right)\left(z-x_{1}\right)}\right) \varphi\left(x_{1}\right) \mathrm{dx}_{1} \quad$.

This is a solution of the first boundary value problem of the first kind in the upper half of the Wplane. The real part in equation (8) is:

$\varphi(x, y)=\frac{1}{\pi} \int_{-\infty}^{+\infty}\left(\frac{y}{\left(x-x_{1}\right)^{2}+y^{2}}\right) \varphi\left(x_{1}\right) \mathrm{dx}_{1} \quad \cdots$

Equation (9) Represents a POISSON's integral formula for the upper half-plane, and for an array of (i+1) electrodes separated by gaps $\mathrm{g}_{1}, \mathrm{~g}_{2}, \ldots \ldots \ldots, \mathrm{g}_{\mathrm{i}}$, equation (9) becomes

$$
\begin{aligned}
& \varphi(x, y)=\left[\varphi_{0} \frac{y}{\pi} \int_{-\infty}^{+\infty}\left(\frac{d x_{1}}{\left(x-x_{1}^{2}\right)+y^{2}}\right) \varphi_{1} \frac{y}{\pi} \int_{g_{1}}^{g_{2}}\left(\frac{d x_{2}}{\left(x-x_{2}^{2}\right)+y^{2}}\right)+\right. \\
& \left.\ldots .+\varphi_{i} \frac{y}{\pi} \int_{g_{i}}^{+\infty}\left(\frac{d x_{i}}{\left(x-x_{i}^{2}\right)+y^{2}}\right)\right] \ldots 1
\end{aligned}
$$

Equation (10) can be solved to give:

$$
\begin{aligned}
& \left(\frac{\varphi_{0}+\varphi_{1}}{2}\right)+\left(\frac{\varphi_{0}-\varphi_{1}}{\pi}\right) \tan ^{-1}\left(\frac{g_{1}-x}{y}\right)+\left(\frac{\varphi_{1}-\varphi_{2}}{\pi}\right) \tan ^{-1}\left(\frac{g_{2}-x}{y}\right)+ \\
& \ldots+\left(\frac{\varphi_{i-1}-\varphi_{i}}{\pi}\right) \tan ^{-1}\left(\frac{g_{i}-x}{y}\right) \ldots 1
\end{aligned}
$$

To find the complex solution $W=\theta+j \psi$ and the field expression, equation (11) is rearranged and written taking into account the following gaps arrangement:

$\tan ^{-1}\left(\frac{g_{l}-x}{y}\right)=\varphi_{l}=\left(\varphi_{l}-\pi / 2\right) .$.

Where $\theta_{l}=\arg \left(z-g_{l}\right)$ is the angle between $\left(z-g_{l}\right)$ and the positive $\mathrm{X}$-axis. Then,

$$
\begin{aligned}
& \varphi(x, y)=\varphi_{i}+\left(\frac{\varphi_{0}-\varphi_{1}}{\pi}\right) \theta_{1}+\ldots \ldots+\left(\frac{\varphi_{i-1}-\varphi_{i}}{\pi}\right) \theta_{i}=\sum_{n=1}^{i} \varphi_{i} \\
& +\left(\frac{\varphi_{n-1}-\varphi_{n}}{\pi}\right) \theta_{n} \quad \ldots
\end{aligned}
$$

And

$\varphi(x, y)=\varphi_{i}+\left(\frac{\varphi_{0}-\varphi_{1}}{\pi}\right) \arg \left(z-g_{1}\right)+\ldots \ldots .+\left(\frac{\varphi_{i-1}-\varphi_{i}}{\pi}\right) \arg$

$\left(z-g_{i}\right)=\sum_{n=1}^{i} \varphi_{i}+\left(\frac{\varphi_{n-1}-\varphi_{n}}{\pi}\right) \operatorname{ard}\left(z-g_{n}\right) \cdots$

Now, equation (14) has the following equivalent Electrical Transformation:

$\left[\varphi(x, y)-\varphi_{i}\right]=\operatorname{Re}\left[\frac{-j}{\pi} \sum_{n=1}^{i}\left(\varphi_{n-1}-\varphi_{n}\right) \ln \left(R_{n}\right)+j \theta_{n}\right]$

When $\mathrm{R}, \theta$, are polar coordinates and

$$
\begin{aligned}
& R_{l}=\left[\left(x^{2}-g_{l}^{2}\right)+y^{2}\right]^{1 / 2} \\
& \theta_{l}=\tan ^{-1}\left[\frac{g_{l}-x}{y}\right]
\end{aligned}
$$

Substituting equation (16) into (15) yields:

$\varphi(x, y)=\sum_{n=1}^{i} \varphi_{i}+\left(\frac{\varphi_{n-1}-\varphi_{n}}{\pi}\right) \theta_{\mathrm{n}} \quad$.

Now from equation 15, 16, and 17 we can deduce Now from equation 15,16 , and 17 we can deduce the complex potential as given by

$W=\varphi+j \psi=\sum_{n=1}^{i} \varphi_{i}+\left(\frac{\varphi_{n-1}-\varphi_{n}}{\pi}\right) \ln \left(\mathrm{z}-\mathrm{g}_{\mathrm{n}}\right) \quad \cdots$

And,

$|\underline{\mathrm{E}}|=\left|-j \frac{\overline{d w}}{d z}\right|=\sum_{n=1}^{i}\left(\frac{\varphi_{n-1}-\varphi_{n}}{\pi}\right)\left(\overline{\frac{1}{z-g_{n}}}\right)=$

$\left|-j \sum_{n=1}^{i}\left(\frac{\varphi_{n-1}-\varphi_{n}}{\pi}\right)\left(\frac{\left(x-g_{n}\right)+j y}{\left(x-g_{n}\right)^{2}+y^{2}}\right)\right| .$. 
Table 1: Testing results

\begin{tabular}{cccccccccc}
\hline Voltage (mV) & \multicolumn{2}{c}{ Current (pA) -110 Degrees C } & \multicolumn{3}{c}{ Current (pA) -130 Degrees C } & \multicolumn{3}{c}{ Current (pA) -150 Degrees C } \\
\cline { 2 - 10 } & Gap 5 & Gap10 & Gap15 & Gap 5 & Gap10 & Gap15 & Gap 5 & Gap10 & Gap15 \\
\hline 50 & 3.025 & 3.147 & 2.795 & 2.708 & 3.398 & 4.432 & 3.834 & 3.896 & 9.412 \\
100 & 3.265 & 3.54 & 3.61 & 3.305 & 3.886 & 6.413 & 4.784 & 4.545 & 17.796 \\
150 & 3.475 & 3.895 & 4.344 & 3.896 & 4.395 & 8.32 & 5.747 & 5.268 & 32.82 \\
200 & 3.602 & 4.266 & 4.826 & 4.42 & 4.88 & 10.375 & 6.705 & 6.042 & 41.73 \\
250 & 3.791 & 4.65 & 5.506 & 4.91 & 5.44 & 13.095 & 7.916 & 6.794 & 45.57 \\
300 & 3.99 & 4.97 & 6.304 & 5.395 & 5.97 & 16.785 & 8.888 & 7.596 & 46.7 \\
350 & 4.16 & 5.265 & 7.059 & 5.999 & 6.46 & 26.2 & 10.013 & 10.395 & 46.73 \\
400 & 4.301 & 5.55 & 7.665 & 7.194 & 6.985 & 34.69 & 11.118 & 11.294 & 46.82 \\
450 & 4.48 & 5.76 & 8.067 & 7.265 & 7.61 & 37.92 & 11.806 & 12.185 & 46.82 \\
500 & 4.625 & 6.065 & 8.162 & 7.746 & 8.27 & 41.46 & 13.045 & 13.323 & 46.82 \\
550 & 4.789 & 6.39 & 8.853 & 8.323 & 8.898 & 44.42 & 14.196 & 14.396 & 46.82 \\
600 & 4.932 & 6.699 & 9.618 & 8.815 & 9.421 & 45.89 & 15.296 & 15.595 & 46.82 \\
650 & 5.15 & 6.979 & 10.935 & 9.398 & 10.221 & 46.35 & 16.65 & 16.946 & 46.82 \\
\hline
\end{tabular}

Then,

$|\underline{\mathrm{E}}|=\left|-j \sum_{n=1}^{i}\left(\frac{\varphi_{n-1}-\varphi_{n}}{\pi}\right)\left(\frac{z-g_{n}}{\left|z-g_{n}\right|^{2}}\right)\right| \cdots$

Equation (20) is resolved to give:

$|\underline{\mathrm{E}}|=\left[\sum_{n=1}^{i}\left[\left(\frac{\varphi_{n-1}-\varphi_{n}}{\pi}\right)\left(\frac{\left(x-g_{n}\right)}{\left(x-g_{n}\right)^{2}+y^{2}}\right)\right]+\right.$
$\left.\left[\left(\frac{\varphi_{n-1}-\varphi_{n}}{\pi}\right)\left(\frac{y}{\left(x-g_{n}\right)^{2}+y^{2}}\right)\right]\right]^{1 / 2} \ldots$

From equation (21):

I. $\mathrm{x}=g_{n}$ :

$|\underline{\mathrm{E}}|=\mathrm{E}_{\mathrm{y}}=\left[\sum_{n=1}^{i}\left(\frac{\varphi_{n-1}-\varphi_{n}}{\pi}\right)^{2}\left(\frac{1}{y}\right)^{2}\right]^{1 / 2}=\left[\sum_{n=1}^{i}\left(\frac{\varphi_{n-1}-\varphi_{n}}{\pi}\right)\left(\frac{1}{y}\right)\right] \ldots$

Here, the field is only dependent on the Y-axis with no gaps realized, it is a bisecting field which varies between $\infty$ and 0 as y takes values between 0 and $\infty$. II. $\mathrm{y}=0$ : $|\underline{\mathrm{E}}|=\mathrm{E}_{\mathrm{x}}=\left[\sum_{n=1}^{i}\left(\frac{\varphi_{n-1}-\varphi_{n}}{\pi}\right)\left(\frac{1}{x-g_{n}}\right)\right]$.

The field in this case has a pole at $\left(\mathrm{x}=g_{n}\right)$ for which equation (23) is infinite.

III. $\mathrm{x}>>g_{n}$

$\mathrm{E}_{\mathrm{x}}=\left[\sum_{n=1}^{i}\left(\frac{\varphi_{n-1}-\varphi_{n}}{\pi}\right)\left(\frac{1}{x}\right)\right]$.

Here the field has total dependency on $\mathrm{x}$ and it is parallel.

IV. $x=0$,

$|\underline{\mathrm{E}}|=\mathrm{E}_{\mathrm{y}}=\left[\sum_{n=1}^{i}\left(\frac{\varphi_{n-1}-\varphi_{n}}{\pi}\right)\left(\frac{1}{y^{2}+g_{n}^{2}}\right)^{1 / 2}\right] \ldots$
Equation (25) describes the field bisecting the gaps and electrodes.

V. $\mathrm{y}>\mathrm{g}_{\mathrm{n}}$

Equation (25) is reduced to (22).

VI. $\mathrm{x}=\mathrm{y}=0$

$|\underline{\mathrm{E}}|=\mathrm{E}_{\text {origin }}=\left[\sum_{n=1}^{i}\left(\frac{\varphi_{n-1}-\varphi_{n}}{\pi}\right)\left(\frac{1}{g_{n}}\right)\right]$.

Here the field is completely controlled by the gaps.

Now by integrating equation (26) and division by

$\varphi_{n-1}-\varphi_{n}=\Delta v_{n}$ we obtain:

$G^{\prime}=\sum_{n=1}^{i}\left(\frac{i_{n}^{\prime}}{\Delta v_{n}}\right)=\frac{\sigma}{\pi} \sum_{n=1}^{i} \ln \left[\left(\frac{y_{k}^{2}}{g_{n}^{2}}\right)^{1 / 2}+\frac{y_{k}}{g_{n}}\right] \cdots(27)$

When $y_{k}$ can be chosen to represent the film thickness.

Using the temperature dependent general equation

$\sigma=\sigma_{0} \exp \left(-\left[\frac{T_{0}}{T}\right]^{0.25}\right) \cdots$

Where $\sigma_{0}, \mathrm{~T}_{0}$ are functions of fermi levels.

Now, substituting equation (28) into (27) gives

$G^{\prime}=\left[\frac{\sigma_{0}}{\pi} \exp \left(\frac{-T_{0}}{T}\right)^{0.25}\right] \sum_{n=1}^{i} \ln \left[\left(\frac{y_{k}^{2}}{g_{n}^{2}}+1\right)^{1 / 2}+\frac{y_{k}}{g_{n}}\right] \ldots$

We can also represent the effect of activation energy through Arrhenius behavior :

$\sigma=A \exp \left(\frac{-E_{a}}{K T}\right) \cdots$

Where $\mathrm{E}_{\mathrm{a}}$ is the activation energy.

Equation (27) become:

$G^{\prime}=\left[\frac{A}{\pi} \exp \left(\frac{-E_{a}}{K T}\right)\right] \sum_{n=1}^{i} \ln \left[\left(\frac{y_{k}^{2}}{g_{n}^{2}}\right)^{1 / 2}+\frac{y_{k}}{g_{n}}\right] \cdots$ 
Equation (31) expresses conductivity in terms of temp, gap width, and film thickness.

\section{RESULTS AND DISCUSSION}

Table 1 shows the testing results of our array sensor, which clearly indicates an increase in the output signal response of the sensor as a function of three main factors:

1. Distance between conducting electrodes (Interdigital separation).

2. Increase in atmospheric temperature.

3. Effect of gas concentration.

These factors can be used to control the behavior of the sensor in terms of the amount and type of adsorbed odors and the rate of desorption. The table also shows that the increase in output signal current is exponential. The mathematical law governing the response of the sensor is affected by the way the Organic Semi conducting layer is sublimed and its thickness and uniformity.

Overall, the obtained characteristics of the tested groups of sensors very much agree with the literature and more proves the response shape of such sensor to be a specific case of power law response, which is a real exponential. Each sensor can be modeled as a group of three sensors grouped together which initiates the need to use pattern recognition and smart classification techniques ${ }^{[14-16]}$.

A suggested neural model would associate the three previously mentioned important parameters, which would implicitly determine gas concentration level and its type. Such a model should allow the process of discrimination between different types of gases and would be able to establish the level of detected gas.

\section{REFERENCES}

1. Carotta, M.C., G. Martinelli, L. Crema, C. Malagù, M. Merli, G. Ghiotti and E. Traversa, 2001. Nanostructured thick film gas sensors for atmospheric pollutant monitoring: Quantitative analysis on field tests. Sensors and Actuators B, 76: 336-342.

2. Hatfield, J.V., J.A. Covington and J.W. Gardner, 2000. GasFETs incorporating conducting polymers as gate materials. Sensors \& Actuators B, 65: 253256.

3. Gardner, J.W., E. Llobet and E.L. Hines, 1999. PSPICE model for resistive gas and odor sensors. Proc. IEE Circuits, Devices and Systems, 146: 101104.

4. Gutierrez-Osuna, R. and N.U. Powar, 2003. Odor mixtures and chemosensory adaptation in gas sensor arrays. Intl. J. Artificial Intelligence Tools, 12: 1-16.
5. Gutierrez-Osuna, R. and A. Gutierrez-Galvez, 2003. Habituation in the KIII olfactory model with chemical sensor arrays. IEEE Trans. on Neural Networks, 14: 1565-1568.

6. Gardner, J.W. and M. Z. Iskandarani, 1992. Effect of electrode geometry on gas sensitivity of lead phthalocynine thin film. Sensors and Actuators B, pp: 133-142.

7. Cole, M., J.W. Gardner, A.W.Y. Lim, P.K. Scivier and J.E. Brignell, 1999. Polymeric resistive bridge gas sensor array driven by a standard cell CMOS current drive chip. Sensors and Actuators B, 58: 518-525.

8. Hines, E.L., E. Llobet and J.W. Gardner, 1999. Electronic noses: A review of signal processing techniques. Proc. IEE Circuits, Devices and Systems, 146: 297-310.

9. Dyer, D.C. and J.W. Gardner, 1997. High precision intelligent interface for a hybrid electronic nose. Sensors and Actuators B, 62: 724-728.

10. Gardner, J.W., E.L. Hines and C. Pang, 1996. Detection of vapours and odours from a multisensor array using pattern recognition: selforganising adaptive resonance techniques. Measurement \& Control, 29: 172-178.

11. Perera, A., T. Sundic, A. Pardo, R. GutierrezOsuna and S. Marco, 2002. A portable electronic nose based on embedded PC technology and GNU/Linux: Hardware, software and applications. IEEE Sensors J., 2: 235-246.

12. Gardner, J.W., H.W. Shin, E.L. Hines and C.S. Dow, 2000. An electronic nose system for monitoring the quality of potable water. Sensors \& Actuators B, 69: 336-341.

13. Gutierrez-Osuna, R. and H.T. Nagle, 1999. A method for evaluating data-preprocessing techniques for odor classification with an array of gas sensors. IEEE Trans. Systems, Man, and Cybernetics B, 29: 626-632.

14. Gutierrez-Osuna, R., A. Gutierrez-Galvez and N.U. Powar, 2003. Transient response analysis for temperature modulated chemoresistors. Sensors and Actuators B: Chemical B, 93: 57-66.

15. Cole, M., J.W. Gardner, D.C. Dyer and P.N. Bartlett, 2001. Low-drift odour and vapour ratiometric resistive elements for analogue CMOS smart sensors. 8th Intl. Symp. Olfaction and the Electronic Nose, 25-30 Mar., Washington DC, USA.

16. Shin, H.W., J.W. Gardner and E.L. Hines, 2000. An electronic nose system to diagnose illness. Sensors \& Actuators B, 70: 19-24. 